\title{
Primordial Excitation and Depletion of the Main Belt
}

\author{
Jean-Marc Petit \\ Centre National de la Recherche Scientifique and Observatoire de la Côte d'Azur \\ (now at Observatoire de Besançon) \\ John Chambers \\ NASA Ames Research Center \\ Fred Franklin \\ Harvard-Smithsonian Center for Astrophysics \\ Makiko Nagasawa \\ Tokyo Institute of Technology
}

\begin{abstract}
The asteroid belt is characterized by a large dynamical excitation, an important mass depletion compared to its original density, and a radial mixing of bodies with different physical and chemical properties. Two mechanisms have been proposed to explain this structure: the sweeping of secular resonances through the belt, and the scattering action by embryos later ejected from the solar system by planetary perturbations. The resonance sweeping is due to the dissipation of the gas-drag-inducing primordial nebula. Resonance sweeping and gas drag are responsible for mass depletion and orbital migration. Embryos are naturally created in the inner solar system during planet formation, producing a strong dynamical excitation. Although both approaches are fairly good at structuring the asteroid belt and depleting it, it is likely that both mechanisms occurred at one time or another. More work is needed to assess the combined effect of the nebula and the excitation by embryos.
\end{abstract}

\section{INTRODUCTION}

The different populations of small bodies (asteroid belt, Edgeworth-Kuiper Belt, irregular satellites, and comets) provide strong constraints about the early evolution of the solar system. The size and chemical and orbital distributions of small bodies, by their large number, carry statistically significant information, while the properties of the planets may be altered by unlikely stochastic events.

In this chapter, we review the main processes that tend to reproduce the observed characteristics of the asteroid belt. The Edgeworth-Kuiper Belt is also of great statistical significance, but the small amount of accurate data prevents discrimination among the models that already exist, and makes any refinement difficult. As for the planets, their detailed dynamical characteristics are not considered here but models must still account for the chemical and isotopic observations.

We now present the most important characteristics of the asteroid belt. In discussing these, we primarily consider asteroids with diameters larger than $50 \mathrm{~km}$ for the following reasons. During the 4.5 G.y. of existence of the solar system, the asteroids have evolved greatly through highvelocity collisions. Collision velocities are typically a few kilometers per second, very often resulting in the complete shattering and disruption of the colliding bodies. Therefore, most of the asteroids one sees today are not primordial, but fragments of larger asteroids destroyed in a collision. Only the largest asteroids retain characteristics that relate to the formation of the asteroid belt and that were not drastically changed by the later evolution. Asteroids with diameters $\mathrm{D}>50 \mathrm{~km}$ have a collisional lifetime of the order of the age of the solar system or longer (Geissler et al., 1996). Most of these are primordial asteroids, i.e., they were already present in the belt at the end of its excitation and mass depletion, after the terrestrial planets were completely formed. The few large bodies that were destroyed generally yielded at most one large fragment (larger than $50 \mathrm{~km}$ ), with mostly unchanged dynamical characteristics, and a swarm of smaller fragments (Tanga et al., 1999). In addition, it is very likely that all the asteroids larger than $50 \mathrm{~km}$ have been discovered; the completeness limit is likely to be about $25 \mathrm{~km}$ (Jedicke and Metcalfe, 1998). So our statistics are not contaminated by observational biases. From Fig. 1a, one can naturally distinguish three zones: the inner belt, with semimajor axis $\mathrm{a}<2.5 \mathrm{AU}(3: 1$ mean motion resonance with Jupiter); the central belt, $2.5<\mathrm{a}<3.28$ AU (2:1 resonance); and the outer belt, beyond $3.28 \mathrm{AU}$. In the outer belt, all asteroids beyond 3.8 AU are in mean-motion resonances with Jupiter. The most striking aspects that one would like to explain with a unitary model are the following:

1. Strong dynamical excitation. The median eccentricity $e_{m}$ and inclination $i_{m}$ respectively are 0.15 and $6^{\circ}$ in the inner belt, 0.14 and $10.7^{\circ}$ in the central belt, and 0.1 and 

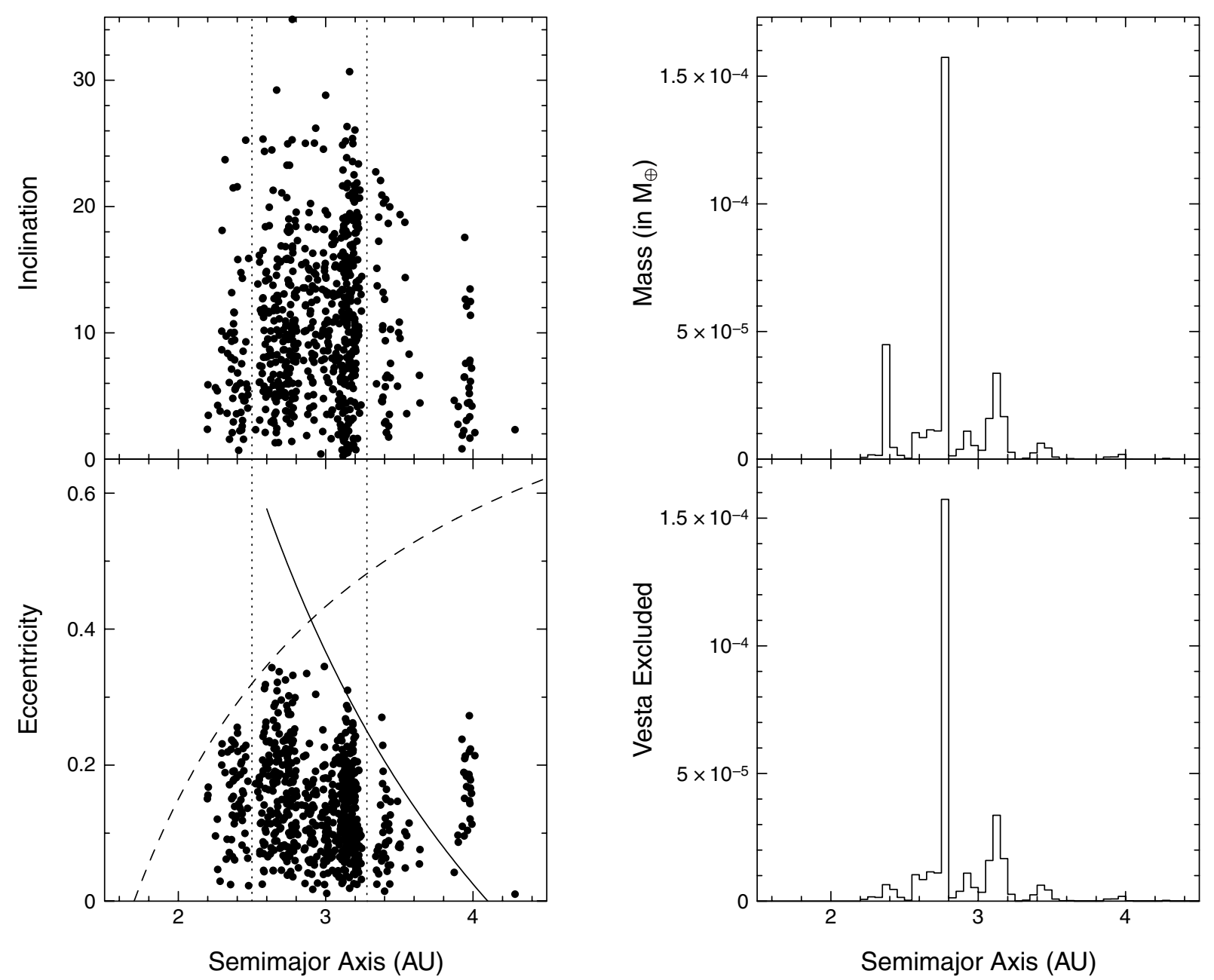

Fig. 1. (a) Osculating inclination (top) and eccentricity (bottom) vs. semimajor axis for the asteroid belt for bodies larger than $50 \mathrm{~km}$ in diameter (solid line: aphelion distance of $4.1 \mathrm{AU}$; dashed line: perihelion distance of $1.7 \mathrm{AU}$ ). (b) Mass distribution of asteroids vs. semimajor axis for all asteroids larger than $50 \mathrm{~km}$ (top), and excluding Vesta (bottom). The dotted lines give the boundaries of the inner belt (right), central belt (middle), and outer belt (right). From Petit et al. (2001).

$12.1^{\circ}$ in the outer belt. In the outer belt, $\mathrm{e}_{\mathrm{m}}$, with the exception of the bodies in the 3:2 and 4:3 resonances, is lower than in the other belts because of the instabilities due to Jupiter that tend to deplete the region above the solid line in Fig. 1a. The eccentricity e and inclination $i$ of the asteroids are much larger than those of the planets in the solar system (with the exception of Mercury and the special case of Pluto), and much larger than would allow accretion. Actually, the absence of bodies at low e $(<0.05)$ and $\mathrm{i}\left(<2^{\circ}\right)$, which would be stable over the age of the solar system (Duncan, 1994; Holman and Murray, 1996), indicates that the primordial excitation in the outer belt has been even larger than in the rest of the asteroid belt, as confirmed by the larger median value of inclination in the former. In the central belt, modeling the existence of (2) Pallas on an orbit with $\mathrm{e}=0.23$ and $\mathrm{i}=34.8^{\circ}$ has only recently been successful (Petit et al., 2001).
2. Large mass depletion. The present total mass of the asteroid belt is estimated to be on the order of $5 \times 10^{-4} \mathrm{M}_{\oplus}$ $\left(\mathrm{M}_{\oplus}=\right.$ Earth mass $)$, namely $10^{3}-10^{4} \times$ smaller than its primordial mass (Lecar and Franklin, 1973). The accretion of the largest asteroids on a timescale comparable with the meteoritic solidification age also implies the primordial existence of at least $100 \times$ more material than at present (Wetherill, 1989). This shows that the important mass deficiency of the asteroid belt is not due to the presence of a gap in the primordial disk, but is the result of some process that occurred after the formation of the asteroids. From Fig. 1b, one sees that the mass deficiency is larger in the inner and outer belts than in the central belt.

3. Radial mixing of asteroid types. The optical properties of the asteroids depend roughly on their distance from the Sun: S-types dominate the inner belt, C-types are the most abundant in the central belt, and P-types dominate in 
the outer belt (with exception of the Trojan population, which is mainly D-type). This radial compositional zoning likely reflects the temperature gradient of the primitive nebula. However, the boundaries between compositional zones are not sharp: Asteroids of different types are mixed over scales of 1 AU (Gradie and Tedesco, 1982). Such mixing is not easily explained by the turbulence of the primitive nebula or by the radial decay of pristine bodies due to gas drag (Ruzmaikina et al., 1989).

The gravitational perturbations arising from all the planets in their current orbits, even acting over times on the order of a billion years, are unable to account for either the range in, or average values of, the e and $i$ of the asteroids, nor for the striking mass depletion of the belt (Duncan et al., 1989). Intense primordial collisional activities have been invoked to explain the large mass deficiencies of the asteroid belt (Chapman and Davis, 1975). Because large bodies cannot be collisionally destroyed, the collisions could have reduced the total mass to its present value only if the original number of large bodies was basically the same as the present one and the original size distribution was very steep (Davis et al., 1979; Wetherill, 1989). However, the survival of the fragile basaltic crust of Vesta is an important argument against a primordial collisional activity significantly more important than the present one (Davis et al., 1994). Therefore it seems that the large mass deficiency of the belt also requires dynamical mechanisms capable of displacing most of the material into unstable regions.

Several mechanisms have been proposed over the last 25 years to explain the dynamical structure of the asteroid belt, with much renewed interest over the last decade. They can be classified in two main categories: those that invoke the sweeping of the belt by resonances of various types, most especially secular resonances, and those that invoke the scattering action of embryos that have later been ejected from the solar system by planetary perturbations. Up to now, these two approaches seem to have ignored each other completely, the first one mostly assuming the dominant dynamical effect of the gaseous primordial nebula, the other one relying on the gravitational perturbations of embryos in a gas-free environment.

The idea that secular resonances could have swept through the asteroid belt as a result of the dissipation of the primordial nebula has been first proposed by Ward et al. (1976). Heppenheimer (1980) and Ward (1981) developed a planar analytic linear model that showed that the current eccentricities of the asteroids could be explained by the passage of two secular resonances through the asteroid belt. Much work has been done during the last decade on the effect of uniform dissipation of the primordial nebula, culminating with the work of Lecar and Franklin (1997) and Franklin and Lecar (2000). More recently, some authors (Nagasawa et al., 2000) have started investigating a nonuniform dissipation of the nebula to circumvent some limitations of the previous approach. All these works are reviewed in section 2 .
The idea that large Jupiter scattered planetesimals (LJSPs, also called large embryos hereafter) of mass comparable to that of Earth could have dynamically heated the asteroid belt was first proposed by Safronov (1979). The existence of large embryos as leftovers from planetary formation is predicted by all the current theories (Fernandez and Ip, 1996). The tilts of the spin axes of Saturn, Uranus, and Neptune support the idea of collisions with massive bodies (about $1 \mathrm{M}_{\oplus}$ ). The most recent work on this hypothesis concluded that it could not solve the problem (Petit et al., 1999; and section 3.1).

Introducing gravitational perturbation from massive bodies, Wetherill (1992) alternatively proposed an endogenic source of excitation, i.e., that the asteroid belt was originally a massive dynamically cold system, which contained about 200 sublunar- to martian-sized embryos among its population. These embryos excited each other by mutual gravitational interactions until they came close to Jupiter and were ejected from the solar system. At the end of this phase, only a small fraction of the original asteroids survived in the stable regions of the belt, on eccentric and inclined orbits. Due to the availability of fast computers and efficient integration algorithms, many simulations have been recently performed on this topic using direct $\mathrm{N}$-body integrations (Wetherill and Chambers, 1997; Chambers and Wetherill, 2001). Extending the disk of embryos into the terrestrial planet region, Chambers and Wetherill (1998) have shown that this approach is actually one of the most promising for the formation of the terrestrial planets. Petit et al. (2001) used these models to study the excitation and depletion of the asteroid belt (section 3 summarizes all these findings).

In section 4, we identify what, in our minds, are the areas that need further investigation and we try to list the particular questions that should be addressed in the coming years.

\section{SWEEPING SECULAR RESONANCES}

This section focuses on the effects of secular resonances, specifically ones that are not fixed in phase space. The term secular resonance applies to commensurabilities between the apsidal or nodal frequencies of an (asteroid's) orbit and one of the similar eigenfrequencies that describe the complete solar system. An important example in the present solar system is one that defines the inner edge of the main belt at a = 2.0 AU (Knežević et al., 1991), which is a narrow region where both the nodal and apsidal frequencies of an orbit (as driven principally by Jupiter) resonate with one of the secular terms (owing to Saturn) in Jupiter's orbit itself.

\subsection{What Is It, Why Is It of Interest?}

We refer the reader to Brower and Clemence (1961) and Nagasawa et al. (2000) for a full derivation of the equations of secular resonance. Here we only give a hint as to what a secular resonance is, and how it is influenced. 
Let $\mathrm{e}_{\mathrm{j}}, \mathrm{i}_{\mathrm{j}}, \bar{\varpi}_{\mathrm{j}}$, and $\Omega_{\mathrm{j}}$ be the eccentricity, inclination, longitude of perihelion, and ascending node of body $\mathrm{j}$. We introduce the canonical variables

$$
\begin{gathered}
\mathrm{h}_{\mathrm{j}}=\mathrm{e}_{\mathrm{j}} \sin \varpi_{\mathrm{j}} \\
\mathrm{k}_{\mathrm{j}}=\mathrm{e}_{\mathrm{j}} \cos \varpi_{\mathrm{j}} \\
\mathrm{p}_{\mathrm{j}}=\tan \mathrm{i}_{\mathrm{j}} \sin \Omega_{\mathrm{j}} \\
\mathrm{q}_{\mathrm{j}}=\tan \mathrm{i}_{\mathrm{j}} \cos \Omega_{\mathrm{j}}
\end{gathered}
$$

Calling $R_{j}$ the perturbing function for body $j$, i.e., the variation from a pure Keplerian two-body Hamiltonian, the time evolutions of these variables are

$$
\begin{aligned}
& \frac{\mathrm{dh}_{\mathrm{j}}}{\mathrm{dt}}=\frac{1}{\mathrm{n}_{\mathrm{j}} \mathrm{a}_{\mathrm{j}}^{2}} \frac{\partial \mathrm{R}_{\mathrm{j}}}{\partial \mathrm{k}_{\mathrm{j}}} \\
& \frac{\mathrm{dk}_{\mathrm{j}}}{\mathrm{dt}}=-\frac{1}{\mathrm{n}_{\mathrm{j}} \mathrm{a}_{\mathrm{j}}^{2}} \frac{\partial \mathrm{R}_{\mathrm{j}}}{\partial \mathrm{h}_{\mathrm{j}}} \\
& \frac{d p_{j}}{d t}=\frac{1}{n_{j} a_{j}^{2}} \frac{\partial R_{j}}{\partial q_{j}} \\
& \frac{\mathrm{dq}_{\mathrm{j}}}{\mathrm{dt}}=-\frac{1}{\mathrm{n}_{\mathrm{j}} \mathrm{a}_{\mathrm{j}}^{2}} \frac{\partial \mathrm{R}_{\mathrm{j}}}{\partial \mathrm{p}_{\mathrm{j}}}
\end{aligned}
$$

In a system with an asteroid, Jupiter, Saturn, and the Sun, assuming $\mathrm{e}_{\text {ast }}, \mathrm{i}_{\text {ast }} \ll 1$, the solutions are

$$
\begin{aligned}
& \mathrm{h}_{\mathrm{ast}}=v \sin (\mathrm{gt}+\beta)+\sum_{\mathrm{j}=1,2} \frac{\mathrm{v}_{\mathrm{j}}}{\mathrm{g}-\mathrm{g}_{\mathrm{j}}} \sin \left(\mathrm{g}_{\mathrm{j}} \mathrm{t}+\beta_{\mathrm{j}}\right) \\
& \mathrm{k}_{\mathrm{ast}}=v \cos (\mathrm{gt}+\beta)+\sum_{\mathrm{j}=1,2} \frac{v_{\mathrm{j}}}{\mathrm{g}-\mathrm{g}_{\mathrm{j}}} \cos \left(\mathrm{g}_{\mathrm{j}} \mathrm{t}+\beta_{\mathrm{j}}\right) \\
& \mathrm{p}_{\mathrm{ast}}=\mu \sin (\mathrm{ft}+\gamma)+\sum_{\mathrm{j}=1,2} \frac{\mathrm{v}_{\mathrm{j}}}{\mathrm{f}-\mathrm{f}_{\mathrm{j}}} \sin \left(\mathrm{f}_{\mathrm{j}} \mathrm{t}+\gamma_{\mathrm{j}}\right) \\
& \mathrm{q}_{\mathrm{ast}}=\mu \cos (\mathrm{ft}+\gamma)+\sum_{\mathrm{j}=1,2} \frac{\mathrm{v}_{\mathrm{j}}}{\mathrm{f}-\mathrm{f}_{\mathrm{j}}} \cos \left(\mathrm{f}_{\mathrm{j}} \mathrm{t}+\gamma_{\mathrm{j}}\right)
\end{aligned}
$$

where $f$ and $g$ are the frequencies of the free oscillations due to gravitational perturbations, while $f_{j}$ and $g_{j}$ are those of the forced oscillations due to Jupiter and Saturn. In the real solar system, they correspond to the eigenfrequency of the whole system. Whenever one of the denominators vanishes, we have what we call a secular resonance. The presence of a massive nebula influences all these frequencies, and may create resonances at various locations in the solar system.

In working out the details of a different secular resonance problem, Ward et al. (1976) suggested that a decaying solar nebula might force these resonances to sweep through the asteroid belt. It was also clear from that paper's mathematical development that both e and i could in principle be markedly elevated during a secular resonance passage that was slower than characteristic apsidal/nodal periods. Heppenheimer (1980) worked out a practical example of e-pumping in the planar nebula case and Ward (1981) treated the broader theoretical topic of secular resonance sweeping. These early efforts led to a fair amount of interest in scanning secular resonances as a naturally occurring means of sculpturing the asteroid belt for many reasons: (1) The effects of secular resonances on the motions of bodies in evolving solar system models can be carried out in a systematic, highly quantitative and partly analytic way. (2) The existence and eventual decay of a solar nebula (which would automatically move these resonances) had already been, and continued to be, established on the basis of very different yet compelling theoretical and observational grounds. (3) The required nebula density was eminently reasonable. To be more precise, the two important secular resonances, labeled $v_{5}$ and $v_{6}$, that arise from the principal terms defining the apsidal motions of Jupiter and Saturn currently lie near semimajor axes, a, of 0.65 and 2.0 AU. These resonances need to be initially located at a > 4 AU to be able to sweep through the asteroid belt and affect it. The minimum mass (Hayashi, 1981) solar nebula model, with volume density given by

$$
\begin{gathered}
\rho=1.4 \times 10^{-9}\left(\frac{\mathrm{r}}{1 \mathrm{AU}}\right)^{-11 / 4} \\
\exp \left[-\left(\frac{\mathrm{z}}{0.047(\mathrm{r} / 1 \mathrm{AU})^{5 / 4}}\right)^{2}\right] \mathrm{g} / \mathrm{cm}^{3}
\end{gathered}
$$

where $\mathrm{r}<36 \mathrm{AU}$ and $(\mathrm{r}, \mathrm{z})$ are the cylindrical coordinates centered at the Sun, has precisely this property (see Fig. 4 of Nagasawa et al., 2000). (4) Estimates of the characteristic timescales required by secular resonance sweeping to generate the observed asteroid distribution from an initial field of low e, i values yield times from (a few) $\times 10^{4}$ to about $10^{5} \mathrm{yr}$ for a uniformly dispersing nebula. This is a plausible value for the e-folding decay time of a T-Taurilike nebula (Kenyon and Hartmann, 1995). Thus the nebula might remain quiescent for $10^{6}-10^{7} \mathrm{yr}$ before it starts to decay in this shorter interval. For nonuniform depletion the decay time can be a few $\times 10^{5} \mathrm{yr}$ and longer (section 2.3). (5) Moving secular resonances potentially can account for features other than the e and i distributions. They can explain both the extreme depopulation in the outer belt and at least contribute mightily to the very considerable mass loss from the inner and central belts. Coupled with the aerodynamic gas drag also characteristic of the nebula, secular resonances can help to diversify the remaining asteroid population so that various taxonomic types can be found at similar semimajor axes. 


\subsection{Uniform Nebula Depletion}

The first nebula models (Heppenheimer, 1980) to investigate secular resonance sweeping were planar, continuous ones with a radial density profile of $\mathrm{r}^{-3 / 2}$ and a time decay, independent of $r$, governed only by $e^{-t / t} 0$. Nonetheless, encouraging results followed: e values were pumped to likely levels, easily reaching 0.3 during nebula dissipation times in the range $10^{4}<\mathrm{t}_{0}<10^{5} \mathrm{yr}$. During these times both $\mathrm{v}_{5}$ and $v_{6}$ would traverse the entire belt, commencing near Jupiter and coming to rest, as the nebula density fell to zero, at their current locations. Passage of two resonances would not only elevate asteroidal e, but also aid in generating a broader range of e values. Should only one secular resonance sweep through the belt, then some other mechanism (e.g., particle close encounters) may be needed to produce a dispersion in e and/or in i, as we shall discuss later. But note (Ward et al., 1976) that the amplitude of e and i depend on phasing of the relevant apsidal/nodal angles. Thus two bodies at the same semimajor axis, feeling the same secular resonance at the same time, may acquire very different e and $i$.

Although these timescales and amplitudes are very satisfactory, a corollary of Ward's (1981) discussion made it clear that the excitation of the $i$ would not be successful if approached in such a simple way. All disks with densities proportional to $\mathrm{r}^{-3 / 2}$, whether or not a gap exists around the orbit of Jupiter (and/or Saturn), will increase the nodal regression of an asteroid's orbit. Since nodes in the belt regress more rapidly than Jupiter's (or more precisely, more rapidly that the value characteristic of the $v_{16}$ resonance) in the absence of a nebula, the needed equality for resonance will never occur. The failure of the $\mathrm{r}^{-3 / 2}$ uniformly decaying nebula to pump the $\mathrm{i}$ has been duly confirmed numerically by Nagasawa et al. (2000).

It is important to recognize that all calculations above have employed the $\mathrm{r}^{-3 / 2}$ density law characteristic of the Hayashi model and supported by Weidenschilling (1977). But this choice of exponent does not agree with the extensive studies by Cameron (1995), who favors an $\mathrm{r}^{-1}$ relation. Bell et al. (1997) propose even shallower gradients, between $\mathrm{r}^{-1}$ and $\mathrm{r}^{-0.5}$, in the asteroid belt. Lemaître and Dubru (1991), assuming a nebula with constant vertical density, examined three examples of the density law, $\mathrm{r}^{-\mathrm{s}}$, with $\mathrm{s}=1$, $3 / 2$, and 2 (and uniform time decay), and found that $\mathrm{s}=1$, Cameron's preferred value, allows $v_{16}$ to sweep through the entire asteroid belt. However, Nagasawa et al. (2000), adopting a more realistic Gaussian vertical profile, found that no nodal resonance would sweep through the entire belt for positive values of $\mathrm{s}$. At present we do not know whether this claim is very general and if a more detailed probing is required. Still, there are grounds for a pessimistic assessment so that, as we shall discuss in section 2.3, "nonuniform" models of nebula dissipation may be the most likely — and possibly the only — way to elevate i throughout the entire belt.
We turn now to the question of mass loss from the belt. The reason for the marked emptiness of the outer belt has bothered dynamicists for several decades (Liou and Malhotra, 1997, and references therein). Ida and Lin (1996) examined the effects of drag combined with mean-motion resonance with Jupiter, and found that this could explain the extreme depletion of the outer belt, provided a minimum mass nebula disapears in $10^{6-7} \mathrm{yr}$. This prompted Lecar and Franklin (1997) to consider drag and secular resonance inasmuch as both are integral properties of any nebula. One needed to evaluate the resulting behavior when the eccentricity reducing drag interacted with the e-enhancing resonances. Such a combined process would promote mass loss via a reduction in the semimajor axes of the asteroids. This paper, still using a two-dimensional Hayashi nebula, showed that e-pumping by secular resonance would empty the outer belt, whether drag was important or not, provided only that nebula removal times were greater than about $10^{4} \mathrm{yr}$, a fact that attests to the rapidity of e-pumping and the efficiency of gravitational scattering of high-e bodies by Jupiter. For bodies in the inner belt, it pointed out that drag would operate on the eccentric orbits of objects several tens of kilometers in radius and spiral them into the inner solar system if the removal time, $\mathrm{t}_{0}$, were greater than about 60,000 yr.

An informal question asked at a past Gordon Conference, wondering if dynamical means could explain why meteorites, apparently originating from the same part of the belt, could have different physical properties, prompted a return to this general problem (Franklin and Lecar, 2000) in order to obtain a more complete statistical sampling. Some results from that paper, again relying on the two-dimensional minimum mass model that included Jupiter and Saturn moving in eccentric, precessing orbits, are contained in Tables 1 and 2 . Table 1 provides the fate in four categories of some 8000 randomly chosen outer belt bodies, lying in four size ranges, as a function of four timescales stretching from 30,000 to $240,000 \mathrm{yr}$. This survey reconfirms that the combined action of secular resonance sweeping and drag readily depopulates the outer belt, but that the "end" for any body depends upon its radius: Drag quickly shoots small objects into the inner belt and the inner solar system, but larger ones, being less affected by drag, are more likely to encounter Jupiter because of their augmented eccentricities. However, some material is transferred from the outer belt into the inner and central belts in most of the examined cases.

Turning now to the inner and central belts, because the two shorter timescales (cases A and B in Table 1) failed to produce more than a token mass reduction in the inner belt, Table 2 concentrates on case $\mathrm{C}$ - hence a dispersal time, $\mathrm{T}_{\mathrm{n}}$, of 120,000 yr - for four different asteroidal radii. Mass loss now becomes considerable, potentially reaching 80 $90 \%$ for all four radii. Increasing $\mathrm{T}_{\mathrm{n}}$ by a factor of 2 would elevate these percentages to $>95 \%$. Beyond Tables 1 and 2, this paper makes the following additional comments on the 
TABLE 1. Fate of bodies of various radii, r, with initial semimajor axes, in the range $3.22<\mathrm{a}<3.85$.

\begin{tabular}{|c|c|c|c|c|}
\hline Case & $\begin{array}{c}\text { Close } \\
\text { Encounter } \\
\text { with Jupiter }\end{array}$ & $\begin{array}{c}\text { Remaining in } \\
\text { Outer Belt }\end{array}$ & $\begin{array}{l}\text { Driven to } \\
\text { Inner Belt }\end{array}$ & $\mathrm{a}<2.1 \mathrm{AU}$ \\
\hline \multicolumn{5}{|c|}{$5<r<10 \mathrm{~km}$} \\
\hline A & 4.6 & 2.4 & 54.4 & 38.6 \\
\hline B & 6.4 & 1.2 & 42.2 & 50.2 \\
\hline $\mathrm{C}$ & 3.6 & 1.0 & 12.8 & 82.6 \\
\hline $\mathrm{D}$ & 1.8 & 0.4 & 0 & 97.8 \\
\hline \multicolumn{5}{|c|}{$10<r<20 \mathrm{~km}$} \\
\hline A & 17.2 & 2.0 & 25.2 & 55.6 \\
\hline B & 22.7 & 1.6 & 23.9 & 51.8 \\
\hline $\mathrm{C}$ & 40.6 & 1.4 & 7.0 & 49.9 \\
\hline D & 27.0 & 0.4 & 1.2 & 71.4 \\
\hline \multicolumn{5}{|c|}{$20<r<50 \mathrm{~km}$} \\
\hline A & 48.2 & 3.4 & 4.0 & 44.4 \\
\hline B & 65.8 & 1.6 & 3.4 & 29.3 \\
\hline $\mathrm{C}$ & 73.9 & 1.6 & 0.7 & 23.7 \\
\hline $\mathrm{D}$ & 80.5 & 0.5 & 0.4 & 18.5 \\
\hline \multicolumn{5}{|c|}{$50<r<100 \mathrm{~km}$} \\
\hline A & 91.1 & 1.4 & 1.8 & 5.6 \\
\hline B & 90.0 & 0.9 & 1.0 & 7.2 \\
\hline $\mathrm{C}$ & 95.9 & 1.3 & 0.2 & 2.6 \\
\hline $\mathrm{D}$ & 97.9 & 0.6 & 0.1 & 1.4 \\
\hline
\end{tabular}

Entries are percentages drawn from a total of about 8000 orbits, evenly divided between the four ranges in $\mathrm{r}$. Labels on columns 2-5 refer to (1) close encounter with Jupiter, (2) and (3) capture into the outer or inner asteroid belts, and (4) passage into the sunward side of the secular resonance $\mathrm{a}$, where $\mathrm{a} \simeq 2.1 \mathrm{AU}$. Cases A-D correspond to nebula (e-folding) dispersal times of 2,500, $5,000,10,000$, and $20,000 \mathrm{P}_{\mathrm{J}} . \mathrm{P}_{\mathrm{J}}$ is the jovian orbital period, $11.862 \mathrm{yr}$. A close encounter with Jupiter means a jovian approach within 10 planetary radii. From Franklin and Lecar (2000).

inner belt for $T_{n}=120,000 \mathrm{yr}$ : (1) Removal of bodies does become increasingly rare when their radii are greater than $800 \mathrm{~km}$, but (2) it is virtually certain for radii less than about $50 \mathrm{~km}$ and (3) this latter limit approximately scales exponentially with $T_{n}$. As is the case for collisional erosion, depletion by gas drag seems to require a steep size distribution. We should also note that it is quite possible for asteroids several hundreds of kilometers in radius [e.g., (4) Vesta] once to have orbited at distances much larger than ones where they are found today.

Finally we return in Fig. 2 to the query raised earlier, whether material from various regions of the belt might be collected at essentially the same semimajor axis. In the heuristic numerical example presented, a body at $\mathrm{a}_{\mathrm{o}}=$ 3.6 $\mathrm{AU}[0.69, \mathrm{a}(\mathrm{J})=1.0]$ and another at $2.9 \mathrm{AU}$ both come to rest at a $=2.73 \mathrm{AU}$, near the center of the inner belt. The outer body "feels" secular resonance at $\mathrm{t}=15,000 \mathrm{P}_{\mathrm{J}}$; its e was increased to 0.35 and semimajor axis consequently reduced by drag. The nebula density has fallen from its
TABLE 2. Fate of bodies of various radii, r, with initial semimajor axes, in the range $2.18<\mathrm{a}<3.12 \mathrm{AU}$.

\begin{tabular}{|c|c|c|c|}
\hline $\mathrm{a}<2.1 \mathrm{AU}$ & $\begin{array}{c}\text { Inner Belt } \\
\text { with e }<0.4\end{array}$ & $\begin{array}{c}\text { Mars } \\
\text { Crosser }\end{array}$ & $\begin{array}{l}\text { Close Encounter } \\
\text { with Jupiter }\end{array}$ \\
\hline \multicolumn{4}{|c|}{$r=60 \mathrm{~km}$} \\
\hline 84.2 & 7.2 & 5.8 & 2.8 \\
\hline \multicolumn{4}{|c|}{$r=120 \mathrm{~km}$} \\
\hline 57.4 & 21.6 & 17.2 & 3.8 \\
\hline \multicolumn{4}{|c|}{$r=240 \mathrm{~km}$} \\
\hline 49.2 & 20.8 & 18.0 & 12.0 \\
\hline \multicolumn{4}{|c|}{$r>1000 \mathrm{~km}$} \\
\hline 0 & 24.0 & 27.8 & 48.2 \\
\hline
\end{tabular}

Nebula dispersal time is $10,000 \mathrm{P}_{\mathrm{J}}$. Entries are percentages based on a total of 2000 orbits, evenly divided between the four ranges in r. Labels on columns 1-4 refer to objects that have (1) passed into the sunward side of the belt; (2) continued in stable orbits in the central or inner belts; (3) also in one of these belts, but with eccentricities that force a crossing of Mars' orbit; and (4) approached Jupiter to within 10 radii and hence no longer move in minor-planet-like orbits. Nearly half the bodies that cross Mars' orbit will cross Earth's as well. Of the 224 bodies in the last two " $r$ " categories that remain in the inner belt, $13 \%$ have proper e in the range $0<\mathrm{e}_{\mathrm{p}}<0.10 ; 26 \%$ from $0.10<\mathrm{e}_{\mathrm{p}}<0.20 ; 38 \%$ from $0.20<\mathrm{e}_{\mathrm{p}}<0.30$, and $23 \%$ with $0.30<\mathrm{e}_{\mathrm{p}}<0.40$. From Franklin and Lecar (2000).

initial value by a factor of about 10 by the time $(t=25,000$ $\mathrm{P}_{\mathrm{J}}$ ) a secular resonance has encountered the second object. It is much less affected by the drag and therefore spirals inward by a lesser amount. Franklin and Lecar (2000) furnish additional examples.

\subsection{Nonuniform Nebula Depletion}

Although the details of nebula depletion are not clear even in the solar system, it is plausible that the dependence of the decay on distance from the central star is not uniform. Several mechanisms have been proposed for the nebula depletion: tidal interaction with a protoplanet ( $\mathrm{Lin}$ and Papaloizou, 1993), photoevaporation by ultraviolet radiation (Shu et al., 1993; Hollenbach et al., 2000), and magnetorotational instability (Balbus and Hawley, 1991; Hawley et al., 1995; Papaloizou and Lin, 1995). Any nebula depletion mechanism in the inner solar system may differ from that in the outer one. When Jupiter starts gas accretion, a gap would open along its orbit. The gap can cover the entire inner solar system and extend to $\sim 15 \mathrm{AU}$ in about $10^{6} \mathrm{yr}$ (Takeuchi et al., 1996). Photoevaporation tends to deplete the outer region (exterior to $10 \mathrm{AU}$ ), where the solar gravitational potential is reduced. Magnetorotational instability tends to develop in the outer regions (outside $20 \mathrm{AU}$ ), since the ionization fraction is high there (Sano et al., 2000). 
Fig. 2. An example showing the ability of scanning secular resonances, by pumping eccentricities and by acting in the presence of drag, to deposit bodies initally at very different semimajor axes, finally at nearly the same one. Crosses correspond to an object with $\mathrm{r}=70 \mathrm{~km}$, triangles with $\mathrm{r}=400 \mathrm{~km}$, and stars with one too large $(r=5000 \mathrm{~km})$ to be affected by drag. Nebula's e-folding decay time is 10,000 jovian periods. Franklin and Lecar (2000) discuss additional cases.

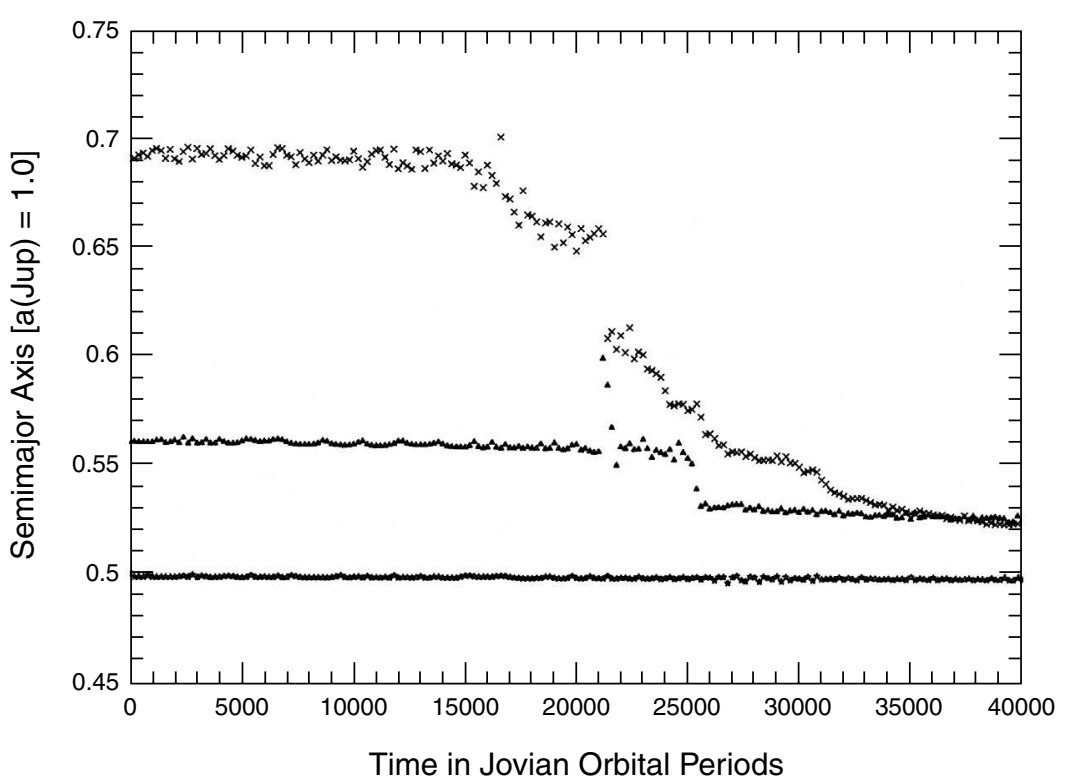

More recently, we have studied the case of nebula depletion caused by Jupiter opening a gap, essentially corresponding to the outwardly migrating edge model. We take the Hayashi minimum mass nebula to begin with. The gap first opens interior to Jupiter's orbit according to $\rho(r)=0$ for $\mathrm{r}_{\text {in }}(\mathrm{t})<\mathrm{r}<5.5 \mathrm{AU}$, the nebula density being unchanged elsewhere. Next, the gap opens outward: $\rho(\mathrm{r})=0$ for $0<$ $\mathrm{r}<\mathrm{r}_{\text {out }}(\mathrm{t})$, where $\mathrm{r}_{\text {out }}$ starts from 5.5 AU and increases. In the model, we include Jupiter and Saturn with their current masses, eccentricities of about 0.048 and 0.054 , and inclination with respect to the invariant plane of 0.0063 and 0.016 radians respectively. Contrary to Nagasawa et al. (2000), we use a nebula whose midplane coincides with the invariant plane of Jupiter and Saturn. However, the nebula midplane is fixed, and the nebula does not respond to the gravitational perturbations of the giant planets. Therefore the $v_{15}$ still exists, although it has a much lower effect than in Nagasawa et al. (2000).

As in the outwardly migrating edge model, this gap formation model excites both e and $i$ all over the asteroid belt and avoids the strong gas drag that leads to rapid inward migration of asteroids with pumped-up e and i. Figure 3 shows the excitation magnitudes of eccentricity $\Delta$ e (Fig. 3a) and inclination $\Delta \tan \mathrm{i}$ (Fig. 3b) due to a single sweeping of the secular resonance, analytically calculated as in Nagasawa et al. (2000), and the location of $v_{15}$ and $v_{16}$ with time. The excitation magnitudes are inversely proportional to the square root of edge migration velocity, $\dot{\mathrm{r}}_{\text {edge }}=-\dot{\mathrm{r}}_{\text {in }}=\dot{\mathrm{r}}_{\text {out }}$ (Ward et al., 1976, Nagasawa et al., 2000). All four resonances pass through the asteroid belt, exciting the eccentricities and inclinations. Note that the strong resonances sweep the asteroid belt when the outer nebula is depleted. The second sweeping of the $v_{5}$ and $v_{15}$ resonances while the edge is between $5 \mathrm{AU}$ and $10 \mathrm{AU}$ (between $5 \times 10^{5}$ and $10 \times 10^{5} \mathrm{yr}$ ) is mainly responsible for the total pumping up of e and $i$. The e and $i$ of asteroids are pumped up to the with the invariant plane of the solar system. 

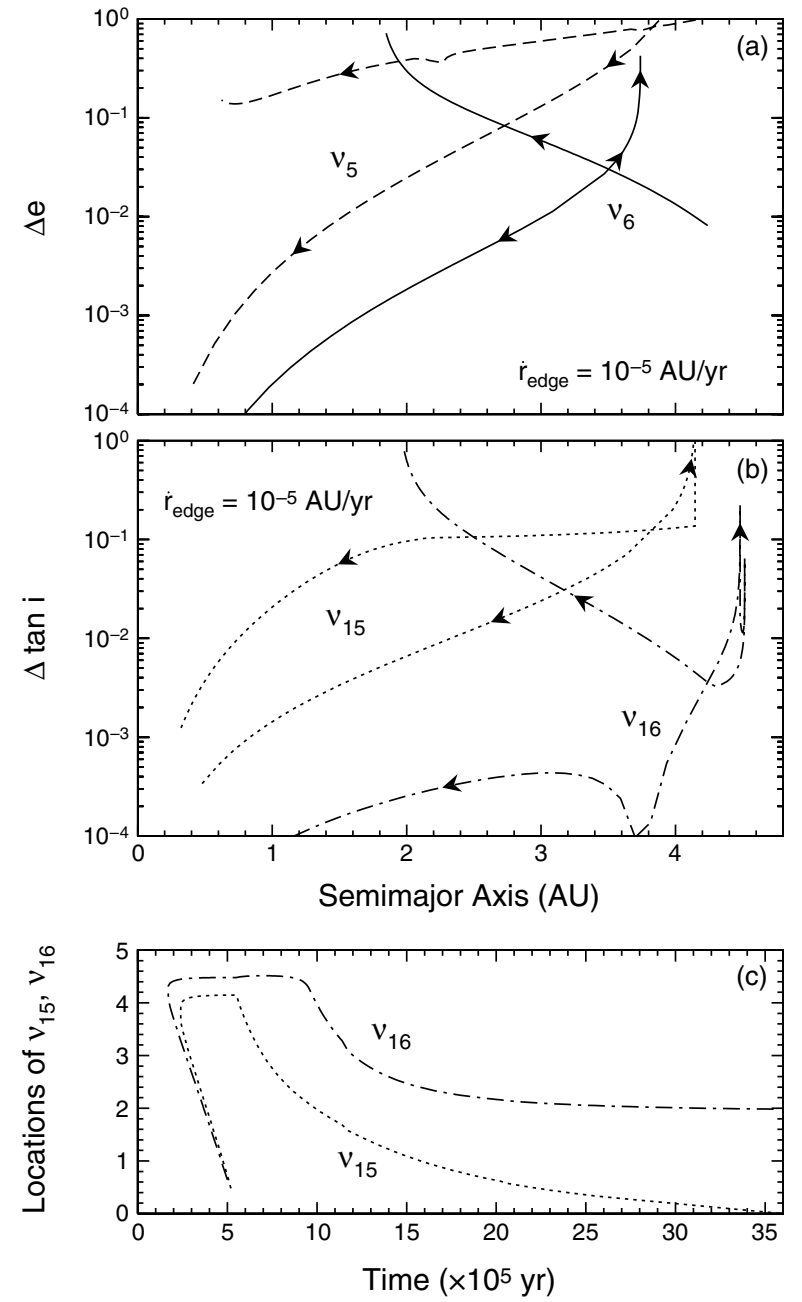

Fig. 3. (a) Changes in eccentricity due to a single sweep of the $v_{5}$ (dashed lines) and $v_{6}$ (solid lines) resonances in the jovian gap formation model. The arrows show the directions of resonances migration. (b) Changes in inclination due to the $v_{15}$ (dotted lines) and $v_{16}$ (dash-dotted lines) resonances. (c) Locations of secular resonances $v_{15}$ and $v_{16}$ vs. time of disk depletion. The paths of $v_{5}$ and $v_{6}$ are almost the same as those of $v_{15}$ and $v_{16}$ respectively. Here the edge velocity $\dot{\mathrm{r}}_{\text {edge }}=10^{-5}$ AU/yr. As the inner edge moves, the resonances migrate in two directions until the inner disk is gone (about $5 \times 10^{5} \mathrm{yr}$ ). After that, the resonances migrate from outside to inside as the outer edge migrates.

observed value in the entire asteroid belt if the nebula is depleted (i.e., the edge moved by $5 \mathrm{AU}$ ) on a timescale longer than $\tau=5 \times 10^{5} \mathrm{yr}$.

The existence and amplitude of the $v_{15}$ resonance depends on the inclination between the nebula midplane and the invariant plane of the system. More work is needed to infer the effect of the interaction between the planets and the nebula gas.

In the case of the outwardly migrating edge model and the jovian gap formation model, the secular resonances pump up e and $\mathrm{i}$ of the asteroids after nebula gas has al- ready been depleted in that region. Therefore, the orbital excitation of asteroids does not lead to rapid inward migration due to strong gas drag even if the nebula depletion time is as long as that inferred from observed T-Tauri stars $\left(10^{6}-10^{7} \mathrm{yr}\right)$. The resultant eccentricities and inclinations remain therefore consistent with those observed.

\section{GRAVITATIONAL PERTURBATION FROM EMBRYOS}

This section focuses on the gravitational effect that embryos in the inner solar system would have on the asteroid belt. We consider two cases, one where large embryos are within a few tenths of an astronomical unit of Jupiter, and thus greatly perturbed by the giant planet, and another one where the embryos are spread over the asteroid belt and the terrestrial planet region.

\subsection{Large Jupiter-Scattered Planetesimals}

A number of works have quantitatively explored the effects of LJSPs on the asteroid belt in the hope of explaining its present structure and providing a constraint on the number, mass, and lifetime of these embryos. Davis et al. (1979), using a gaslike model, estimated that five $1-\mathrm{M}_{\oplus}$ LJSPs, crossing the belt during 3 m.y., could provide the required eccentricities. Ip (1987) was the first to attempt to follow the dynamical evolution of LJSPs and asteroids. For this purpose, he used a Monte Carlo code based on the method of Arnold (1965) to account for the statistical effects of close encounters, but neglecting distant perturbations and resonant phenomena, as well as the mutual encounters among the LJSPs. He found that the LJSPs have dynamical lifetimes too short to be efficient asteroidal perturbers; only if Jupiter were smaller, i.e., one-tenth of its present mass for $\sim 10$ m.y., could the LJSPs' action be more important. In particular, 50 LJSPs, each of mass $3 \times$ $10^{27} \mathrm{~g}$, could excite the asteroid belt in this approximation. Wetherill (1989), also using Monte Carlo simulations but including the mutual encounters among LJSPs, contradicted the conclusions of $I p$ (1987). He found that, even in the case of a full-sized Jupiter, a LJSP may decrease its eccentricity due to an encounter with another LJSP, isolating itself in the asteroid belt, safe from Jupiter encounters. The isolated body would then have a sufficiently long lifetime to excite the asteroid belt by gravitational scattering. In this case, only $10 \%$ of the asteroids would survive more than 700 m.y., and they would show a similar distribution of e and $i$ to that observed. Moreover, the gravitational scattering would also provide an important mixing in the radial distribution of asteroidal compositional types.

Using the modern tools of celestial mechanics, Petit et al. (1999) revisited this question. They first studied the dynamical evolution of large embryos initially distributed regularly in the range $\mathrm{a} \in[4.0,4.8] \mathrm{AU}$, with low eccentricity $(0.01)$ and inclination $\left(<1^{\circ}\right)$ moving under the gravitational perturbation of Jupiter in its current orbit, and with its current 
mass. Only $15 \%$ of the embryos survived longer than $1 \mathrm{~m} . \mathrm{y}$. in low-eccentricity stable orbits, never encountering Jupiter. In a second set, they let the mass of Jupiter grow linearly with time from 5 to $15 \mathrm{M}_{\oplus}$ over $10 \mathrm{~m}$.y. During this time, only $2 \%$ of the embryos were ejected. At this point, Jupiter should accrete the surrounding gas very rapidly and reach its present mass of more than $300 \mathrm{M}_{\oplus}$ in a very short time (Pollack et al., 1996). After this event, the following evolution would be statistically equivalent to that of the previous case.

Building upon these results, Petit et al. (1999) determined the dynamical excitation and mass loss of the asteroid belt by a Monte Carlo method and direct integration of test particles. In this model, the asteroid belt shows very little excitation between 2 and $3 \mathrm{AU}$, and a rather large excitation beyond $3 \mathrm{AU}$. The excitation exhibits a marked gradient, which is not seen in the actual belt. A very important point is the very low inclination excitation, barely reaching $3^{\circ}$, when an equipartition of energy would imply an inclination on the order of $10^{\circ}$ in the outer belt. Finally, the mass depletion is very small, only a few percent. This is because the embryos are ejected by Jupiter very quickly, and do not get a chance to cross the inner belt. In the case of a growing Jupiter, the results are similar, since most of the embryos do not move until Jupiter reaches its current mass.

The only way to solve this problem is to have scattered embryos in long-lived orbits in the main belt. Due to mutual gravitational interactions, some LJSPs could decrease their eccentricity, isolating themselves in the main asteroid belt, and therefore avoiding close encounters with Jupiter. Petit et al. performed integrations of a system composed of the present Jupiter and five embryos of $1 \mathrm{M}_{\oplus}$. They found only $2 \%$ of the cases producing an embryo decoupled from Jupiter that (1) crossed the entire asteroid belt, (2) did not affect the region of the inner planets, and (3) was in a region dynamically unstable on a moderately long timescale, leaving the embryo time to excite the asteroids but eventually allowing it to escape. The dynamical excitation of the asteroids was then very large, and the radial displacement also quite large, reaching more than $1 \mathrm{AU}$. The depletion of test particles was complete after about 50 m.y.

Even though these last results are encouraging, they are very unlikely, and it is not clear how the unstability of the embryo and its ejection would affect the inner solar system and the terrestrial planets.

\subsection{Endogenic Dynamical Excitation and Mass Depletion}

We now consider the case where the planetary embryos formed in the asteroid belt region. It is widely believed that the early stages of the formation of the terrestrial planets were characterized by runaway growth, in which large solid bodies grew rapidly while small bodies grew more slowly or were themselves accreted (Wetherill and Stewart, 1989; Kokubo and Ida, 2000). After $10^{5}-10^{6} \mathrm{yr}$, when runaway growth ceased, much of the solid material in the terrestrial- planet region had accreted into "planetary embryos" with masses comparable to the Moon or Mars (Wetherill and Stewart, 1993; Weidenschilling et al., 1997). In the currently-favored "core formation" model for the formation of Jupiter and Saturn (Pollack et al., 1996; Ikoma et al., 2000), runaway growth also occurred in the region between 5 and $10 \mathrm{AU}$ from the Sun, producing embryos of 2-15 $\mathrm{M}_{\oplus}$ (Lissauer, 1987; Inaba and Wetherill, 2001). The largest of these objects were sufficiently massive to accrete large atmospheres directly from the gas component of the Sun's protoplanetary nebula.

The formation of planetary embryos in both the inner and outer solar system makes it plausible that embryos also formed in the region now occupied by the main asteroid belt. Numerical simulations (Wetherill and Stewart, 1993; Weidenschilling and Davis, 2001) suggest that in the absence of external perturbations, embryos would have formed in the belt within $\sim 10^{6} \mathrm{yr}$. If this was the case, the absence of planetary embryos or fully formed planets in the modern belt can be viewed as an additional aspect of the great depletion of solid material from the belt that has occurred since the time when the nebula existed.

More interestingly, the existence of planetary embryos in the belt actually provides a mechanism for producing the mass depletion. Wetherill (1992) first proposed and examined this mechanism, which involves a combination of gravitational perturbations by embryos and orbital resonances. The main belt currently contains a number of powerful mean-motion and secular resonances associated with the giant planets. An asteroid in some of these resonances develops an unstable orbit on timescales of $\sim 10^{6} \mathrm{yr}$ (Gladman et al., 1997). As a result, the asteroid's orbital eccentricity undergoes large changes until the asteroid either falls into the Sun or passes close to Jupiter and is gravitationally scattered out of the solar system. Today these resonances are associated with the Kirkwood gaps in the belt, while the regions between the resonances contain large numbers of asteroids with apparently stable orbits. At the time when the planets were accreting, asteroids and embryos in the resonances would have been removed rapidly. However, more asteroids and embryos would have been scattered into the resonances by close encounters with other embryos. Thus a continuous stream of material was perturbed into the resonances and removed from the asteroid belt.

Wetherill (1992) found that a combination of resonances and embryo perturbations was sufficient to remove all embryos from the belt in half of the cases he studied. More recent N-body integrations suggest that the belt would be cleared of embryos in two-thirds of the cases (Chambers and Wetherill, 2001). The same mechanism would also have removed many asteroids from the belt. In fact, small bodies were lost preferentially since "dynamical friction" with the larger embryos tended to increase the eccentricities of the former more than the latter. Objects with large eccentricities were more likely to be removed since the resonances occupy a larger fraction of the belt for eccentric orbits, and these orbits are also more likely to be planet-crossing. 


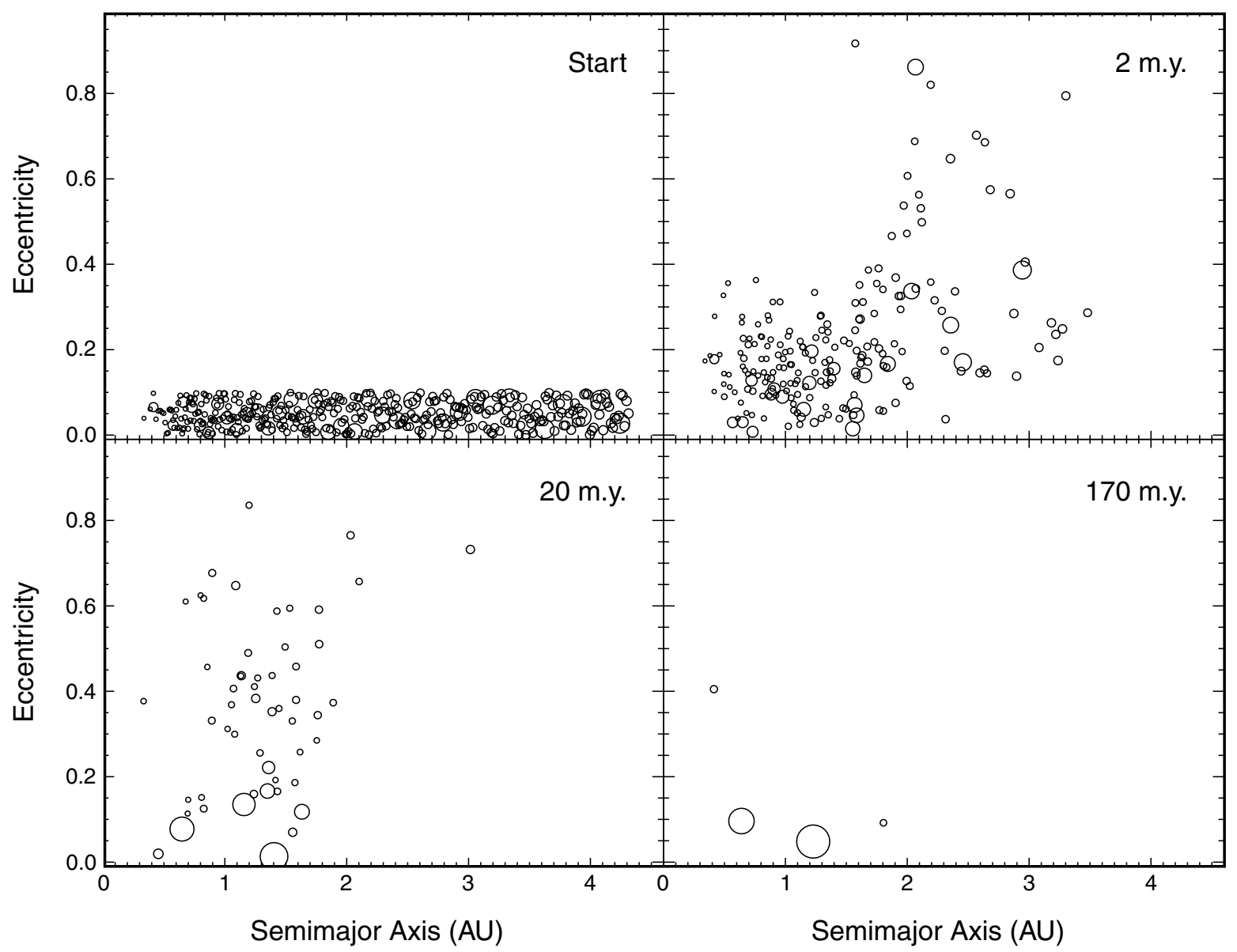

Fig. 4. Orbital evolution of 385 planetary embryos in the terrestrial planet and asteroid regions. Symbol radius is proportional to radius of the embryo. Jupiter and Saturn are included starting on orbits with an eccentricity of 0.1 .

It is probable that $~ 99 \%$ of the original population of asteroids was lost (Petit et al., 2001), so that the observed asteroids are a small group of lucky survivors from a much larger primordial population. Even these objects will have been scattered a number of times by close encounters with embryos. As a result, the observed asteroids typically have large e and $i$, and their orbital distribution essentially fills the volume of phase space that has been stable over the age of the solar system. In addition, if an asteroid's spectral class reflects the original location in the nebula at which it formed, gravitational scattering by embryos explains why the different asteroid classes are partially mixed in terms of heliocentric distance. Petit et al. (2001) found a typical radial displacement of $\sim 0.5 \mathrm{AU}$. In addition, close encounters with embryos in the belt may have tidally stripped the mantles from many small differentiated bodies, leaving intact metalrich cores that survive today as M-type asteroids (Asphaug et al., 2000).

Figure 4 shows the embryo-clearing mechanism in action in an N-body integration. The simulation begins with a disk containing 385 embryos spread across the inner solar system. In addition, fully formed Jupiter and Saturn are present, starting at their current heliocentric distances. Within
$1 \mathrm{~m} . \mathrm{y}$., the resonances increase the eccentricities of embryos in the main belt and material starts to be removed. After 20 m.y., hardly any embryos remain in the belt. Conversely, many embryos remain in the terrestrial region, where powerful resonances are absent, and eventually these accrete to form a system of four terrestrial planets. Such rapid clearing of material from the main belt may help explain why the primordial crust of (4) Vesta has avoided disruption due to large impacts since it was formed in the first few miillion years of the solar system.

One conceptual difficulty with the embryo-clearing mechanism is how the last embryo was removed from the asteroid belt. Once this body had scattered all the other embryos into unstable resonances it could have become trapped on a stable orbit with no mechanism to remove it from the belt. In fact, the last embryo probably collided with or was scattered into a resonance by an embryo in the terrestrial-planet region (Chambers and Wetherill, 2001). Terrestrial embryos would also have cleared many other embryos and asteroids from the inner belt, and this places strong constraints on the masses and orbital evolution of embryos in the region now occupied by Mars (Petit et al., 2001). 
The clearing of mass from the belt affected the orbits of the giant planets, since these bodies ultimately provided the changes in energy and angular momentum needed to eject asteroids and embryos from the belt or cause them to hit the Sun. In response, Jupiter's orbit migrated inward, while the orbits of both Jupiter and Saturn became more circular (Chambers and Wetherill, 2001). Hence, Jupiter originally lay further from the Sun than today, and the giant planets had more eccentric orbits in the past (for this reason the giants initially had $\mathrm{e}=0.1$ in the simulation shown in Fig. 4). The strength of the main-belt resonances depends sensitively on the eccentricities of the giant planets: Doubling e of Jupiter and Saturn increases the flux of material from the belt by a factor of $\sim 10$ (Chambers and Wetherill, 2001). Hence the formation times and early eccentricities of the giant planets determined when most of the primordial mass was removed from the asteroid belt. This in turn affects how much asteroidal material was accreted by the terrestrial planets that were forming at the same time (Morbidelli et al., 2000).

In summary, the formation of planetary embryos in the asteroid belt provides a plausible and efficient mechanism for depleting much of the mass that originally existed in this region of the protoplanetary nebula. It is likely that this process would leave behind a population of asteroid-sized bodies with orbital and taxanomic distributions similar to the observed ones.

\section{CONCLUDING REMARKS AND FUTURE WORK}

In summary, we note that the belt could have been dynamically excited and cleared by a combination of the process discussed in section 3.2 and resonance sweeping, provided that embryos formed quickly before resonance sweeping had removed much material. Both mechanisms do a good job at exciting the eccentricities and inclinations of the asteroids, and removing most of the mass. The sweeping of secular resonance has trouble with the radial mixing since it would tend to produce a fairly homogeneous mixture instead of the overlapping zoning seen today. The gravitational perturbation by planetary embryos may not have occurred if it was the case that Jupiter formed before runaway growth took place in the solar system (Kortenkamp and Wetherill, 2000) as a result, for example, of disk instability (Boss, 2000). As we have implied earlier, the case for secular resonance sweeping does have some unfinished business. Models based on nonuniform nebula depletion clearly excite both e and i, but do not lead to a major decrease in the main belt's population. On the other hand, although uniform depletion can excite e and depopulate the belt, a clearer demonstration is needed as to the range of conditions under which it can excite the i. Perturbation by embryos provides a mechanism to deliver water (and other volatile elements) to Earth, but not necessarily to the other terrestrial planets, by letting an embryo from the outer belt collide with the forming Earth (Morbidelli et al., 2000).
What future developments would be worthwhile? It seems a fair comment that processes naturally arising during the early stages of the solar system may account for many of the properties of the asteroid distribution. But it is also fair to remark that studies to date have proceeded in a rather piecemeal fashion. Astronomers are now approaching the stage when a more comprehensive, integrated discussion is desirable. Such a study must combine the previous mechanisms and include considerations such as (1) gravitational perturbation from planetary embryos with a collective response of the disk of asteroids (as in planetary rings, or in the Kuiper Belt; Ward and Hahn, 1998) and in the presence of gas drag — this would probably not directly affect the embryos, but certainly the asteroids; (2) possible ranges of giant planet migration; and (3) limits imposed by timescales set by giant and terrestrial planet formation.

Another specific question of interest is how, in the sweeping resonance and the endogenic excitation mechanisms, the 2:1 mean motion resonance with Jupiter can be such a dividing line between the low density of the central and inner belts, and the much lower density of the outer belt. Nesvorný and Morbidelli (1998) have shown that chaotic regions in the outer belt are far more abundant than previously noted, thanks primarily to weak three-body meanmotion resonances. What is not presently known is whether the chaos is sufficient to lead to widespread instability, consequently emptying the region in times less than the solar system's age. If chaos does so prevail, then the paucity of objects in the outer belt (but only in the outer belt) can no longer be used as clear evidence favoring any other ejection mechanism.

We hope that this review will help motivate further interpretive studies of the asteroid belt and so keep pace with the rapid advance of observational knowledge, and at the same time promote additional work on the EdgeworthKuiper Belt (Hahn and Malhotra, 1999; Petit et al., 1999) to determine which of those processes that have been applied to the asteroid belt may also prove fruitful there.

\section{REFERENCES}

Arnold J. R. (1965) The origin of meteorites as small bodies. III. General considerations. Astrophys. J., 141, 1548-1556.

Asphaug E., Bottke W. F. Jr., Morbidelli A., and Petit J-M. (2000) Tides versus collisions in the primordial main belt (abstract). Bull. Am. Astron. Soc., 32, 1100.

Balbus S. A. and Hawley J. F. (1991) A powerful local shear instability in weakly magnetized disks. I - Linear analysis. II Nonlinear evolution. Astrophys. J., 376, 214-233.

Bell K. R., Cassen P. M., Klahr H. H., and Henning Th. (1997) The structure and appearance of protostellar accretion disks: Limits on disk flaring. Astrophys. J., 486, 372-387.

Boss A. P. (2000) Possible rapid gas giant planet formation in the solar nebula and other protoplanetary disks. Astron. J. Lett., 536, L101-L104.

Cameron A. G. W. (1995) The first ten million years of the solar nebula. Meteoritics, 30, 133-161.

Chambers J. E. and Wetherill G. W. (1998) Making the terrestrial 
planets: N-body integrations of planetary embryos in three dimensions. Icarus, 136, 304-327.

Chambers J. E. and Wetherill G. W. (2001) Planets in the asteroid belt. Meteoritics \& Planet. Sci., 36, 381-400.

Chapman C. R. and Davis D. R. (1975) Asteroid collisional evolution: Evidence for a much larger early population. Science, 190, 553-556.

Davis D. R., Chapman C. R., Greenberg R., Weidenschilling S. J., and Harris A. W. (1979) Collisional evolution of asteroids, populations, rotations and velocities. In Asteroids (T. Gehrels, ed.), pp. 528-557. Univ. of Arizona, Tucson.

Davis D. R., Ryan E. V., and Farinella P. (1994) Asteroid collisional evolution: Results from current scaling algorithms. Planet. Space Sci., 42, 599-610.

Duncan M. J. (1994) Orbital stability and the structure of the solar system. In Circumstellar Dust Disks and Planet Formation (R. Ferlet and A. Vidal-Madjar, eds.), pp. 245-255. Editions Frontières.

Duncan M. J., Quinn T., and Tremaine S. (1989) The long-term evolution of orbits in the solar system: A mapping approach. Icarus, 82, 402-418.

Fernandez J. A. and Ip W. H. (1996) Orbital expansion and resonant trapping during the late accretion stages of the outer planets. Planet. Space Sci., 44, 431-443.

Franklin F. A. and Lecar M. (2000) On the transport of bodies within and from the asteroid belt. Meteoritics \& Planet. Sci., 35, 331-340.

Geissler P., Petit J. M., Durda D., Greenberg R., Bottke W., and Nolan M. (1996) Erosion and ejecta redistribution on 243 Ida and its moon. Icarus, 120, 140-157.

Gladman B. J., Migliorini F., Morbidelli A., Zappalà V., Michel P., Cellion A., Froeschlé Ch., Levison F., Bailey M., and Duncan M. (1997) Dynamical lifetimes of objects injected into asteroid belt resonances. Science, 277, 197-201.

Gradie J. C. and Tedesco E. F. (1982) Compositional structure of the asteroid belt. Science, 216, 1405-1407.

Hahn J. and Malhotra R. (1999) Orbital evolution of planets embedded in a planetesimal disk. Astron. J., 117, 3041-3053.

Hawley J. F., Gammie C. F., and Balbus S. A. (1995) Local threedimensional magnetohydrodynamic simulations of accretion disks. Astrophys. J., 440, 742-763.

Hayashi C. (1981) Structure of the solar nebula, growth and decay of magnetic fields and effects of magnetic and turbulent viscosities on the nebula. Prog. Theor. Phys. Suppl., 70, 35-53.

Heppenheimer T. A. (1980) Secular resonances and the origin of the eccentricities of Mars and the asteroids. Icarus, 41, 76-88.

Hollenbach D. J., Yorke H. W., and Johnstone D. (2000) Disk dispersal around young stars. In Protostars and Planets IV (V. Mannings et al., eds.), pp. 401-428. Univ. of Arizona, Tucson.

Holman M. J. and Murray N. W. (1996) Chaos in high-order mean motion resonances in the outer asteroid belt. Astron. J., 112, 1278-1293.

Ida S. and Lin D. N. C. (1996) Long term gas drag effects on the structure of the asteroid belt and its implications for the solar nebula. Astron. J., 112, 1239-1246.

Ikoma M., Nakazawa K., and Emori H. (2000) Formation of giant planets: Dependences on core accretion rate and grain opacity. Astrophys. J., 537, 1013-1025.

Inaba S. and Wetherill G. W. (2001) Formation of Jupiter: Core accretion model with fragmentation (abstract). In Lunar and Planetary Science XXXII, Abstract \#1384. Lunar and Planetary Institute, Houston (CD-ROM).

Ip W. H. (1987) Gravitational stirring of the asteroid belt by Jupiter zone bodies. Gerl. Beitr. Geophys., 96, 44-51.

Jedicke R. and Metcalfe T. S. (1998) The orbital and absolute magnitude distributions of main belt asteroids. Icarus, 131, 245-260.

Kenyon S. and Hartmann L. (1995) Pre-main sequence evolution in the Taurus-Auriga molecular cloud. Astrophys. J. Supp. Series, 101, 117-171.

Knežević Z., Milani A., Farinella P., Froeschlé Ch., and Froeschlé C. (1991) Secular resonances from 2 to $50 \mathrm{AU}$. Icarus, 93, 316-330.

Kokubo E. and Ida S. (2000) Formation of protoplanets from planetesimals in the solar nebula. Icarus, 143, 15-27.

Kortenkamp S. J. and Wetherill G. W. (2000) Terrestrial planet and asteroid formation in the presence of giant planets. Icarus, 143, 60-73.

Lecar M. and Franklin F. A. (1973) On the original distribution of the asteroids. I. Icarus, 20, 422-436.

Lecar M. and Franklin F. A. (1997) The solar nebula, secular resonances, gas drag, and the asteroid belt. Icarus, 129, 134-146.

Lemaître A. and Dubru P. (1991) Secular resonances in the primitive solar nebula. Cel. Mech. Dyn. Astron., 52, 57-78.

Lin D. N. C. and Papaloizou J. C. B. (1993) On the tidal interaction between protostellar disks and companions. In Protostars and Planets III (E. H. Levy and J. I. Lunine, eds.), pp. 749835. Univ. of Arizona, Tucson.

Liou J. C. and Malhotra R. (1997) Depletion of the outer asteroid belt. Science, 275, 375-377.

Lissauer J. (1987) Timescales for planetary accretion and the structure of the protoplanetary disk. Icarus, 69, 249-265.

Morbidelli A., Chambers J., Lunine J. I., Petit J-M., Robert F., Valsecchi G. B., and Cyr K. E. (2000) Source regions and time scales for the delivery of water to Earth. Meteoritics \& Planet. Sci., 35, 1309-1320.

Nagasawa M., Tanaka H., and Ida S. (2000) Orbital evolution of asteroids during depletion of the solar nebula. Astron. J., 119, 1480-1497.

Nesvorný D. and Morbidelli A. (1998) Three-body mean motion resonances and the chaotic structure of the asteroid belt. Astron. J., 116, 3029-3037.

Papaloizou J. C. B. and Lin D. N. C. (1995) Theory of accretion disks I: Angular momentum transport processes. Annu. Rev. Astron. Astrophys., 33, 505-540.

Petit J-M., Morbidelli A., and Valsecchi G. (1999) Large scattered planetesimals and the excitation of the small body belts. Icarus, 141, 367-387.

Petit J-M., Morbidelli A., and Chambers J. E. (2001) The primordial excitation and clearing of the asteroid belt. Icarus, 153, 338-347.

Pollack J. B., Hubickyj O., Bodenheimer P., Lissauer J. J., Podolak M., and Greenzweig Y. (1996) Formation of the giant planets by concurrent accretion of solids and gas. Icarus, 124, 62-85.

Ruzmaikina T. V., Safronov V. S., and Weidenschilling S. J. (1989) Radial mixing of material in the asteroidal zone. In Asteroids II (R. P. Binzel et al, eds.), pp. 681-700. Univ. of Arizona, Tucson.

Safronov V. S. (1979) On the origin of asteroids. In Asteroids (T. Gehrels, ed.), pp. 975-993. Univ. of Arizona, Tucson.

Sano T., Miyama S. M., Umebayashi T., and Nakano T. (2000) Magnetorotational instability in protoplanetary disks. II. Ionization state and unstable regions. Astrophys. J., 543, 486-501.

Shu F. H., Johnstone D., and Hollenbach D. (1993) Photoevaporation of the solar nebula and the formation of the giant planets. Icarus, 106, 92-101.

Takeuchi T., Miyama S. M., and Lin D. N. C. (1996) Gap forma- 
tion in protoplanetary disks. Astrophys. J., 460, 832-847.

Tanga P., Cellino A., Michel P., Zappalà V., Paolicchi P., and Dell'Oro A. (1999) On the size distribution of asteroid families: The role of geometry. Icarus, 141, 65-78.

Ward W. R. (1981) Solar nebula dispersal and the stability of the planetary system. I. Scanning secular resonance theory. Icarus, 47, 234-264.

Ward W. R. and Hahn J. M. (1998) Dynamics of the trans-Neptune region: Apsidal waves in the Kuiper belt. Astron. J., 116, 489498.

Ward W. R., Colombo G., and Franklin F. A. (1976) Secular resonance, solar spin down, and the orbit of Mercury. Icarus, 28, 441-452.

Weidenschilling S. J. (1977) The distribution of mass in the planetary system and solar nebula. Astrophys. Space Sci., 51, 153158.

Weidenschilling S. J. and Davis D. R. (2001) Planetary accretion: The first 5 million years (abstract). In Lunar and Planetary Science XXXII, Abstract \#1894. Lunar and Planetary Institute, Houston (CD-ROM).
Weidenschilling S. J., Spaute D., Davis D. R., Marzari F., and Ohtsuki K. (1997) Accretional evolution of a planetesimal swarm. 2: The terrestrial zone. Icarus, 128, 429-455.

Wetherill G. W. (1989) Origin of the asteroid belt. In Asteroids II (R. P. Binzel et al., eds.), pp. 661-680. Univ. of Arizona, Tucson.

Wetherill G. W. (1992) An alternative model for the formation of the asteroids. Icarus, 100, 307-325.

Wetherill G. W. and Chambers J. E. (1997) Numerical integration study of primordial clearing of the asteroid belt (abstract). In Lunar and Planetary Science XXVIII, p. 1547. Lunar and Planetary Institute, Houston.

Wetherill G. W. and Stewart G. R. (1989) Accumulation of a swarm of small planetesimals. Icarus, 77, 350-357.

Wetherill G. W. and Stewart G. R. (1993) Formation of planetary embryos: Effects of fragmentation, low relative velocity, and independent variation of eccentricity and inclination. Icarus, 106, 190-209. 

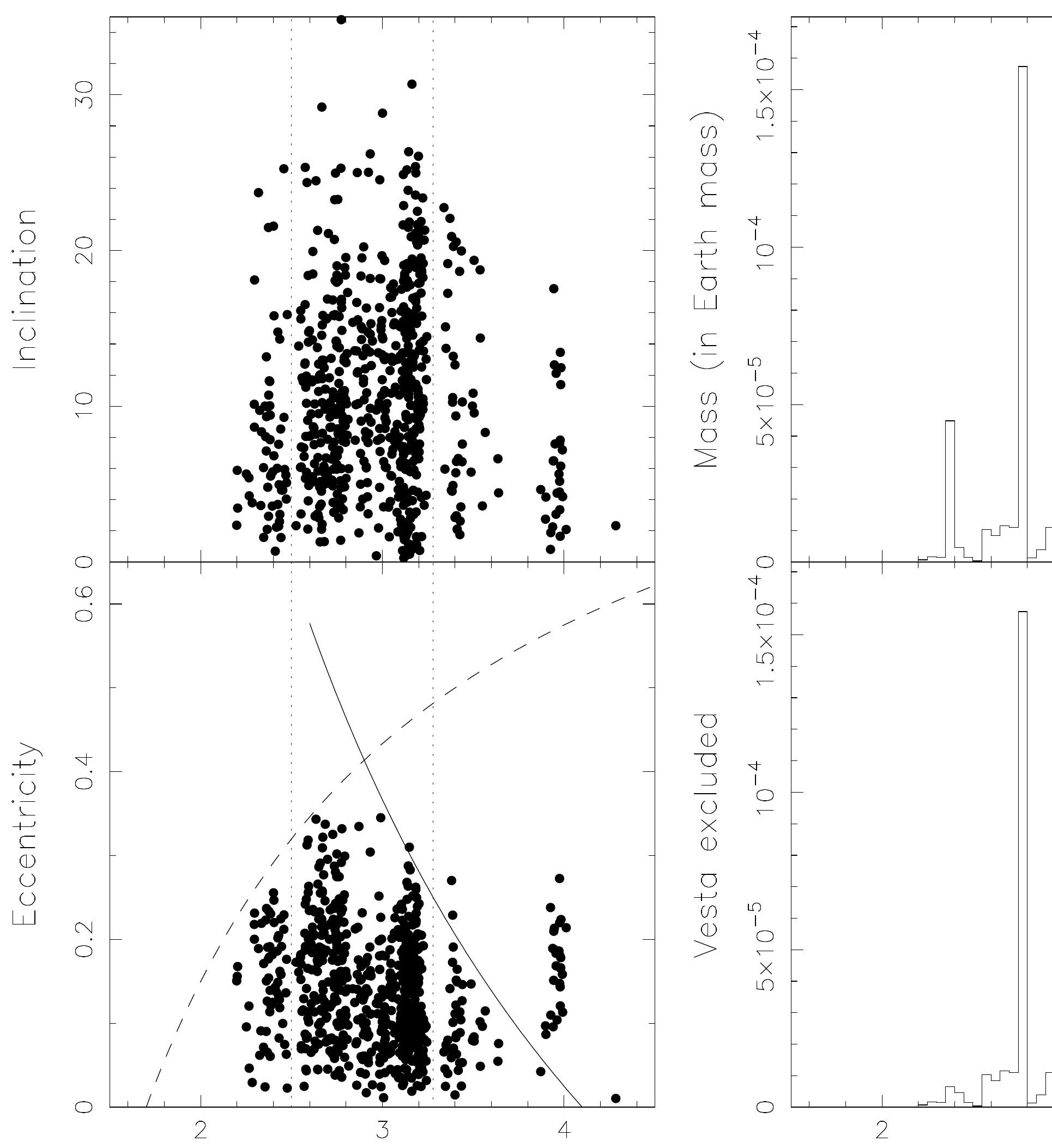

Semi-major axis (AU)

Semi-maj 


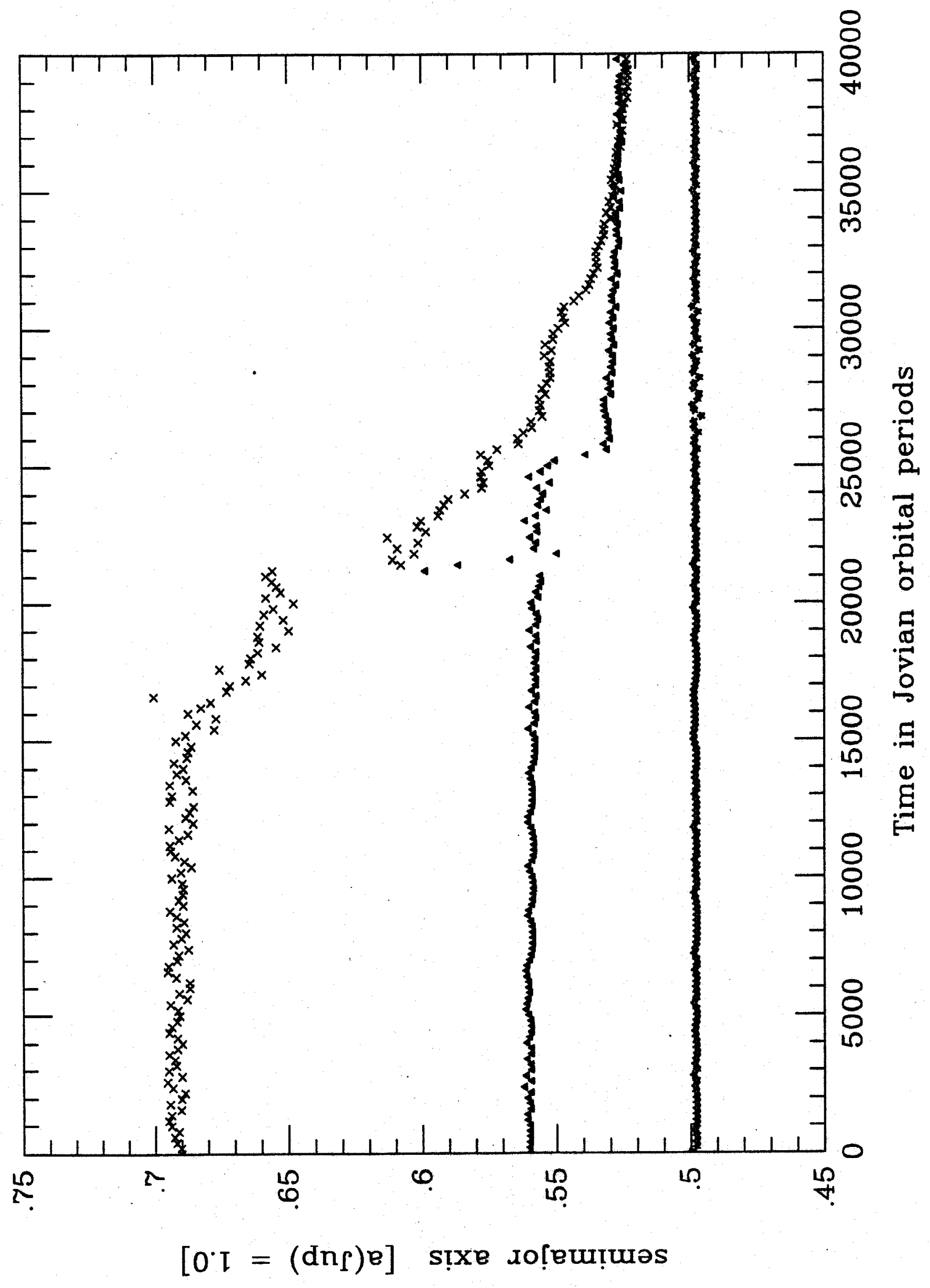



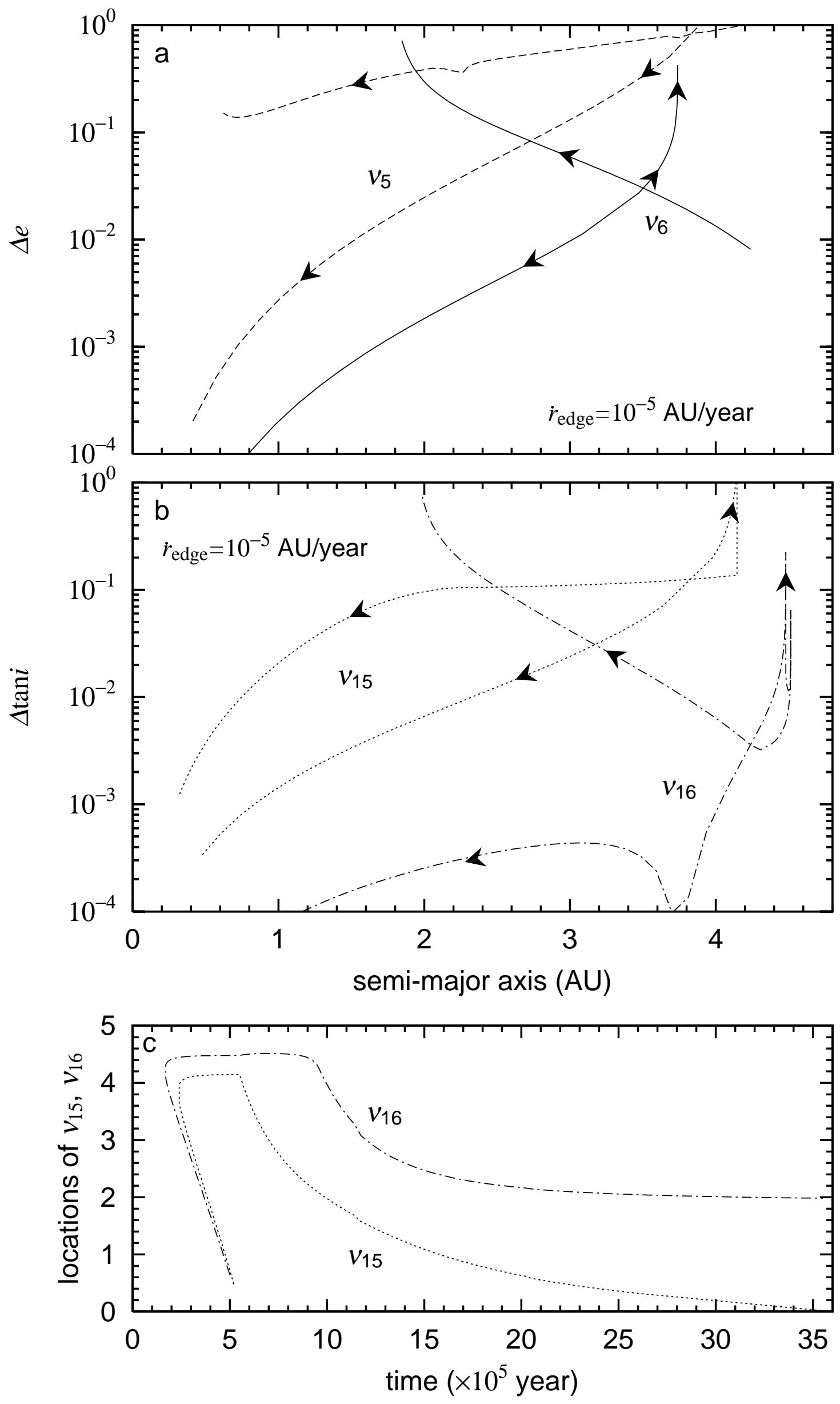
$(\cap \forall)$ strXe doleut-turos

$(\cap V)$ sixe roleut-tutos

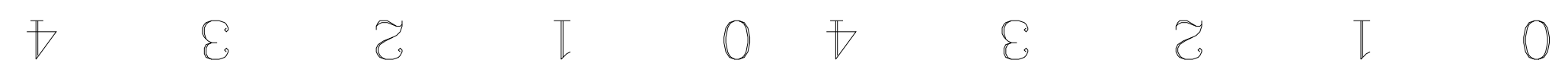

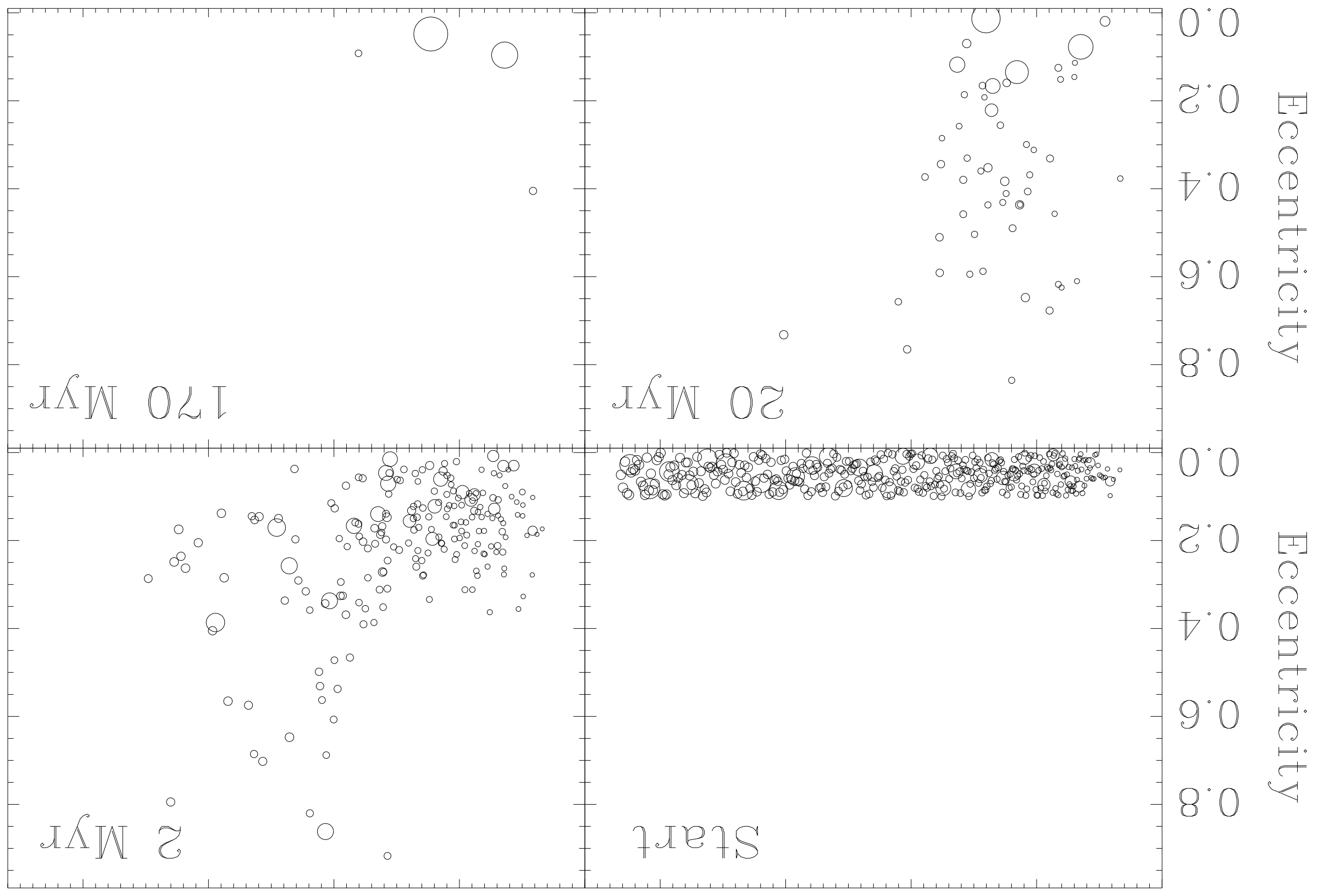

